\title{
Intestine epithelial cell-Derived Extracellular Vesicles alleviate Inflammation Induced by Clostridioides Difficile TcdB through the Activity of TGF- $\beta 1$
}

\author{
Shuangshuang Wan ${ }^{1}$, Guangzhong Song ${ }^{1}$, Hui Hu ${ }^{1}$, Yaqing Xu ${ }^{1}$, Peng Zeng ${ }^{1}$, Shan Lin ${ }^{1}$, Jun Yang ${ }^{1}$, Jinqin Jiang ${ }^{1}$, Xiaojun Song ${ }^{2}$, \\ Dazhi Jin ${ }^{\text {* }}$ \\ ${ }^{1}$ Department of laboratory Medicine, Hangzhou Medical College, Hangzhou, 310053, China. \\ ${ }^{2}$ Centre of Laboratory Medicine, People’s Hospital of Hangzhou Medical College, Zhejiang Provincial People’s Hospital, Hangzhou, Zhejiang, \\ 310014, China.
}

*Corresponding Author: Dazhi Jin, Department of laboratory Medicine, Hangzhou Medical College. No. 481 Binwen Rd, Hangzhou, Zhejiang, 310053, China.

\section{Received Date: October 10, 2021; Accepted Date: November 11 2021; Published Date: November 17, 2021}

Citation: Shuangshuang Wan, Guangzhong Song, Hui Hu, Yaqing Xu, Peng Zeng, et al. (2021). Intestine Epithelial cell-Derived Extracellular Vesicles alleviate Inflammation induced by Clostridioides difficile TcdB THROUGH the Activity of TGF- $\beta 1$, J. Immunology and Inflammation Diseases Therapy. 4(1); DOI:10.31579/2637-8876/018

Copyright: () 2021 Dazhi Jin. This is an open-access article distributed under the terms of The Creative Commons Attribution License, which permits unrestricted use, distribution, and reproduction in any medium, provided the original author and source are credited.

\section{Abstract}

Background: Clostridioides difficile infection (CDI) has been primarily associated with the toxin B (TcdB), which can activate the intestinal immune system and lead to pathological damage. Even though the biological functions of intestine epithelial cell- derived extracellular vesicles (I-Evs) have been well documented, the role of I-Evs in the process of CDI is still unknown.

Results: We isolated I-Evs ranging from 100-200 $\mathrm{nm}$ in mean diameter, with a density of 1.09-1.17 g/mL. These I-Evs expressed the extracellular vesicle-associated specific surface markers, CD63 and TSG101. In vitro, $50 \mu \mathrm{g}$ I-Evs decreased the expression of IL-6, TNF- $\boldsymbol{\beta}$, IL-1 $\beta$, and IL-22 in MC38 induced by $0.8 \mathrm{ng} / \mathrm{mL}$ C. difficile TcdB, and increased expression of TGF- $\beta 1$. In vivo, I-Evs also promoted regulatory $\mathrm{T}$ cell induction, which improved inflammation of mice up to $80 \%$ relative to $\mathrm{C}$. difficile TcdB infected mice, depending on the TGF- $\beta 1$ signal pathway.

Conclusion: Our study firstly demonstrated that I-Evs originated from intestine epithelial cells is potentially a novel treatment endogenous candidate to effectively reduce the local infection induced by $\mathrm{C}$. difficile TcdB.

Keywords: extracellular vesicles; Clostridioides difficile; TGF- $\beta 1$; TcdB; regulatory T cells; inflammatory cytokines; immunotherapy 36

\section{Background}

In recent decades, with the excessive application of broad-spectrum antibiotics, diseases related to intestinal flora disorders have precipitously increased. Clostridioides difficile (C. difficile) is one of the main pathogens leading to antibiotic-associated diarrhoea and hospitalacquired infections in the United States and other developed countries [1]. Toxin A (TcdA) and B (TcdB) are the major pathogenic factors leading to diarrhoea, pseudomembranous colitis, toxic megacolon, and other intestinal symptoms [2]. The mechanism lies in the inactivation in the host epithelial cells of proteins from the Rho family of GTPases-including Rho, Rac, or Cdc42 by glycosylation, and upregulation of a series of proinflammatory cytokines such as interleukin IL-1, IL-6, and TNF- $\alpha$ [3]. Meanwhile, toxins recruit neutrophils and other inflammatory immune cells to induce intestinal mucosal cell apoptosis, necrosis, shedding, and increased permeability, triggering a widespread loss of intestinal barrier function, and initiating imbalance of flora and intestinal epithelial damage. According to the American Infectious Society, and the European Society of Clinical Microbiological Infections, in addition to other practical guidelines, oral metronidazole or vancomycin are the best methods to treat Clostridioides difficile infection (CDI) [4]. In addition, some new narrow-spectrum antibiotics such as fidaxomicin [5] and rifaximin have little impact on the intestinal flora and reduce the risk of drug resistance. In recent years, a number of immune-based agents [6] have entered clinical trials, and however their efficacy needs to be further validated. Faecal microbiota transplantation (FMT) has been recognised in the United States as an optional treatment method to restore normal intestinal flora and prevent recurrent attacks. However, a meta-analysis of randomised clinical trials in 2019 showed that the cure rate of FMT was only $76.1 \%$. Furthermore, there are still many unanswered questions about FMT, including the optimal timing, preparation methods, and the 
patients who are likely to benefit most from this procedure. As its standard protocol is relatively complicated and involves approval of ethical reviews, FMT has not yet been widely used in China. Extracellular vesicles (Evs) are small vesicle-like substances secreted by cells, which possess various biological activities when released outside of the cell. They have a diameter ranging from approximately $30 \mathrm{~nm}$ to $1 \mu \mathrm{m}$, and are generally classified into exosomes, microvesicles, and apoptotic bodies based on their size, biogenesis, and mechanism of secretion [7]. It is difficult to determine the functional differences between these three types of Evs, due to the lack of specific markers with which to distinguish them. Although once thought to be cellular debris, Evs are now recognized as vital vehicles involved in the communication between cells. Research has confirmed that Evs contain a wide range of biologically active components, and their corresponding functions depend on the source tissue or cell type. Evs also exist in body fluids such as serum, alveolar lavage fluid, and breast milk, carry messenger RNAs, microRNAs, and DNA $[8,9]$; this suggests potential applications as biomarkers for the diagnosis of diseases, as part of a liquid biopsy technology [10]. Recently, it has been reported that Evs can be designed to function as effective carriers in the treatment of various diseases, including in the delivery of long non-coding RNAs [11, 12]. In addition, Evs play a significant therapeutic role in regulating complex intracellular pathways in certain diseases, such as inflammatory bowel disease (IBD) [13, 14], and osteoarthritis [15]. Furthermore, it has been discovered that Evs derived from mesenchymal stem cells possess important immunomodulatory effects in areas such as neurodegenerative diseases, ageing, and inflammation [16-18]. Previously, we have reported that CD8 $\alpha+C D 11 \mathrm{c}+$ Evs derived from lungs reduce the allergic reaction of asthmatic mice through TGF- $\beta 1$ and IL-10, thereby maintaining the immune balance of the respiratory tract [19]. In the context of the recent outbreaks of COVID-19 around the world, mesenchymal stem cells and their Evs could be used as potential drug candidates for the treatment of severe cases, mainly through the induction of anti-inflammatory macrophages, regulatory T and B cells, and regulatory dendritic cells [20]. Strikingly, infection with TcdB-producing strains alone, but not TcdA+B- strains, can cause severe CDI symptoms [21]. Our work using purified C. difficile $\mathrm{TcdB}$, together with cell lines and mice, confirmed that TcdB can induce expression of the inflammatory genes IL-6, TNF- $\boldsymbol{\beta}$, IL-22, and IL-1 $\boldsymbol{\beta}$, and upregulation of TGF- $\square 1$ in vitro. Intestine epithelial cell-derived extracellular vesicles (I-Evs) rescue this phenomenon in vivo by inducing proliferation of regulatory $\mathrm{T}$ cells, dependent on TGF- $\beta 1$ and the corresponding downstream molecules Smad2/3. Here, we studied the role of I-Evs on inflammation induced by C. difficile TcdB and evaluated biological functions of I-Evs in alleviating pathological damage led by CDI in mice.

\section{Results}

Isolation and identification of intestine epithelial cell-derived extracellular vesicles We used electron microscopy to visualise the morphology of the purified I-Evs; combined with Nanoparticle tracking analysis this showed that the isolated I-Evs had a mean diameter of 100$200 \mathrm{~nm}$ (Figure 1A, B). To further explore the I-Evs, sucrose density gradient centrifugation was used to detect the density range of I-Evs, which was $1.09-1.17 \mathrm{~g} / \mathrm{mL}$ (Figure 1C). Protein analysis identified that I-Evs were positive for universal surface markers of extracellular vesicles, including CD63 and TSG101, and the intestinal epithelial cell-specific protein A33, but negative for GRP94, as detected by western blot (Figure 1D).

A
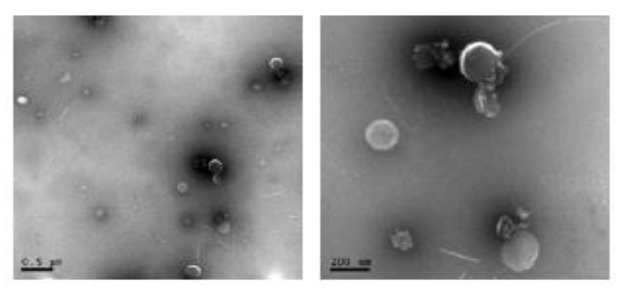

$\begin{array}{lllllllll}1.07 & 1.09 & 1.11 & 1.13 & 1.15 & 1.17 & 1.19 & 1.21 & \text { Density }\end{array}$

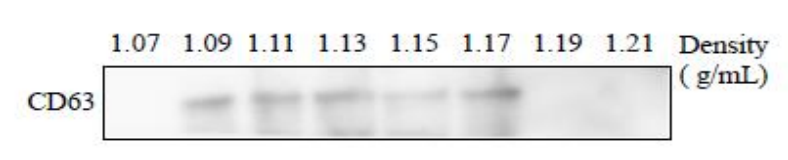

\section{C}

B

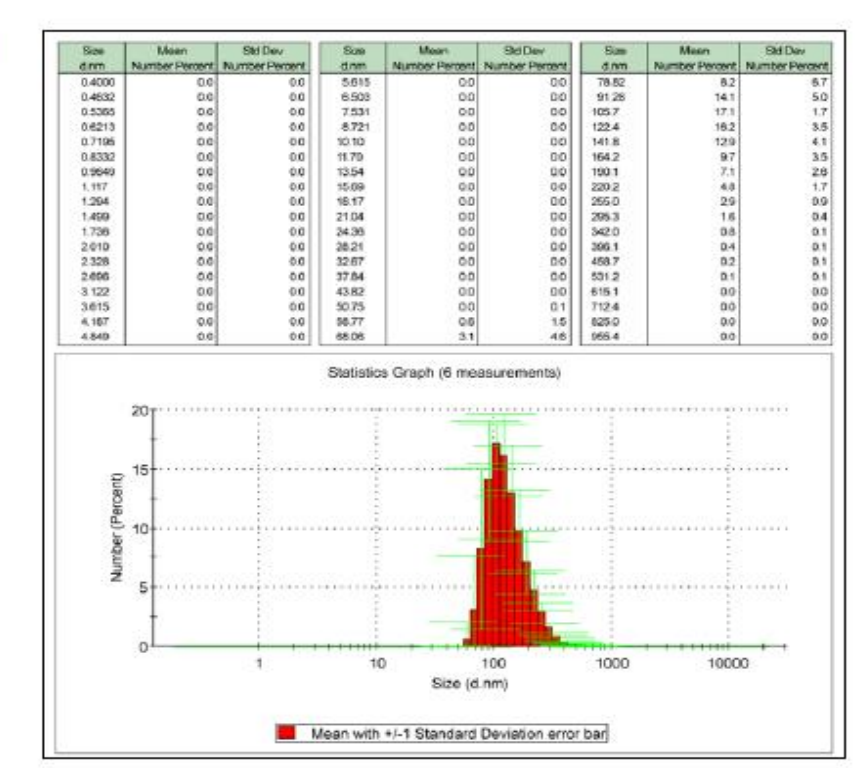

\section{$\mathbf{B}$}


In addition, high levels of TGF- $\beta 1$ were expressed in I- Evs, implying a role in immunoregulation. The results showed that we successfully isolated and identified I-Evs. I-Evs attenuated the down-regulation of TGF- $\boldsymbol{\beta} 1$ induced by purified C. difficile TcdB in vitro Real-time PCR results showed that, compared to the control group, the expression of proinflammatory genes (IL-6, TNF- $\boldsymbol{\beta}$, IL-1 $\beta$, and IL-22) was increased in the $0.4 \mathrm{ng} / \mathrm{mL}$ C. difficile TcdB group, but significantly decreased in the $0.8 \mathrm{ng} / \mathrm{mL}$ I-Evs group. In contrast, the expression of the antiinflammatory genes TGF- $\boldsymbol{\beta} 1$ and IL-10 was statistically increased in the I-Evs group compared to the TcdB groups (Figure 2A).

A
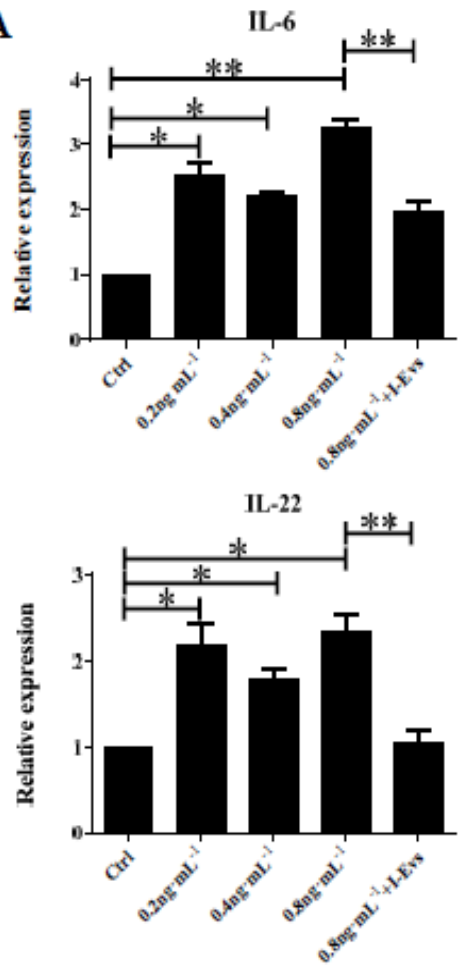

B

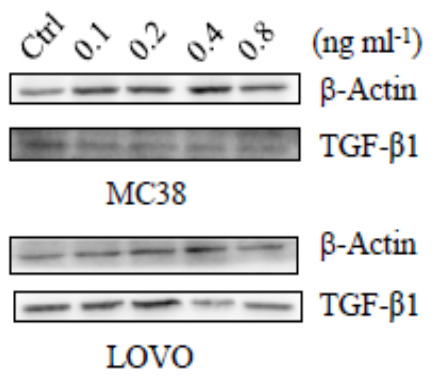

Western blot results showed that protein levels of the immunosuppressive cytokine TGF- $\boldsymbol{\beta} 1$ were decreased in MC38 murine colon carcinoma cells, and LOVO human colon carcinoma cells, stimulated by C. difficile TcdB (Figure 2B). The concentration of C. difficile TcdB used was $0.1 \mathrm{ng} / \mathrm{mL}$, $0.2 \mathrm{ng} / \mathrm{mL}, 0.4 \mathrm{ng} / \mathrm{mL}$, or $0.8 \mathrm{ng} / \mathrm{mL}$. This decrease could be rescued by I-Evs when TcdB concentration was $0.4 \mathrm{ng} / \mathrm{mL}$ (Figure 2C). Altogether, these results indicate that the I-Evs containing TGF- $\beta 1$ had anti- Inf ammatory effects in vitro.
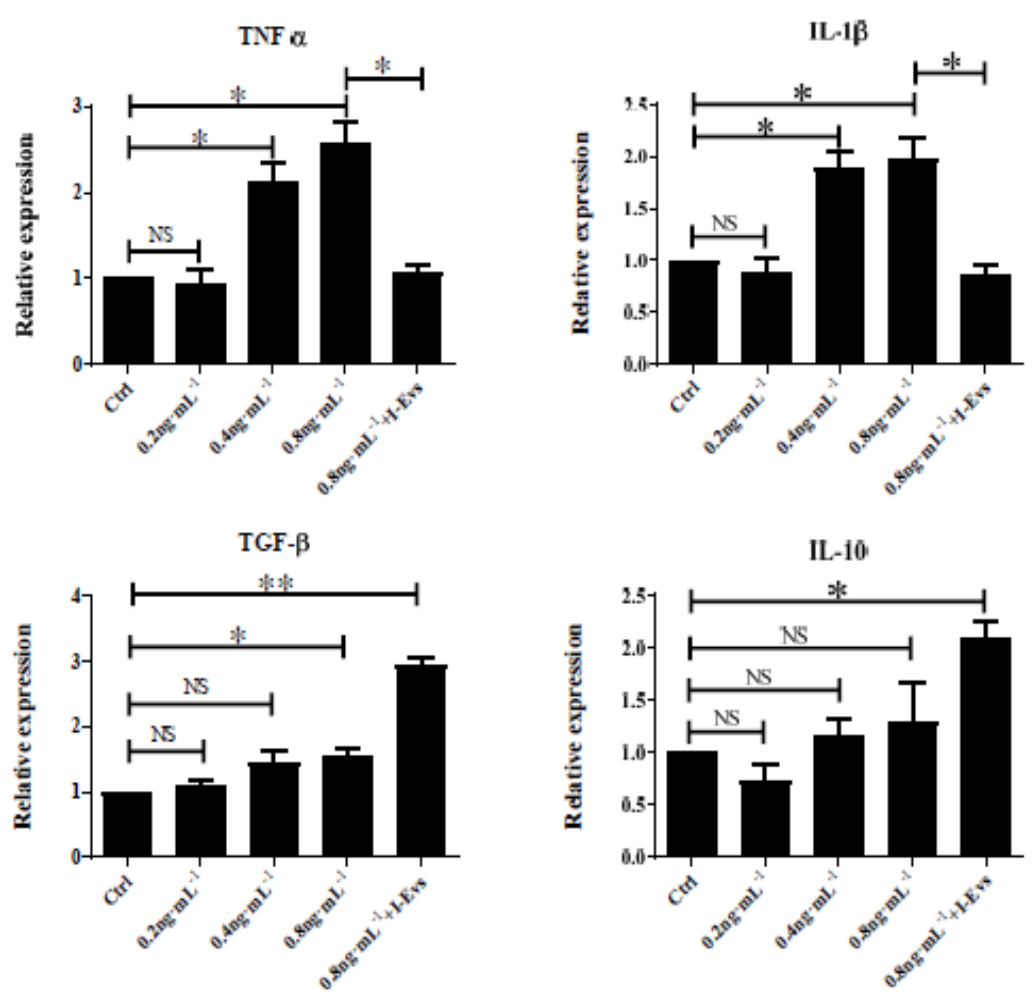

C

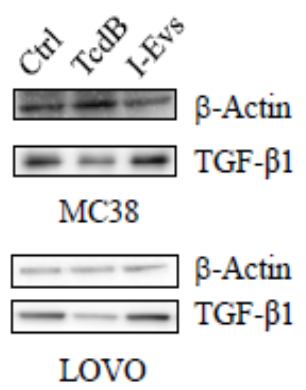

Figure 2: I-Evs attenuated the downregulation of TGF- $\beta 1$ induced by purified $C$. difficile TcdB in vitro. (A) MC38 cells were exposed to different concentrations of $T c d B(0.2 \mathrm{ng} / \mathrm{mL}, 0.4 \mathrm{ng} / \mathrm{mL}$, or $0.8 \mathrm{ng} / \mathrm{mL})$ for $5 \mathrm{~h}$, or simultaneously treated with $50 \mu \mathrm{g}$ I-Evs. Real-time PCR was used to detect gene expression levels of $I L-6, T N F-\beta, I L-1 \beta, I L-22, T G F-\beta 1$, and $I L-10$. (B) MC38 and LOVO cells were stimulated with C. difficile TcdB (0.1 $\mathrm{ng} / \mathrm{mL}, 0.2 \mathrm{ng} / \mathrm{mL}, 0.4 \mathrm{ng} / \mathrm{mL}$, or $0.8 \mathrm{ng} / \mathrm{mL}$ ), before cell lysates were analysed by western blot. (C) Similarly, cells were treated with $C$. difficile TcdB and I-Evs, and the TGF- $\beta 1$ protein levels analysed by western blot. All data were verified by three independent experiments. $P$ values were calculated by one-way analysis of variance (ANOVA), versus control conditions (*: $P<0.05$, **: $P<0.01$, ***: $P<0.001$, NS: not significant).

\section{I-Evs alleviate C. difficile TcdB-induced local colon inflammation in mice}

Intestinal epithelial damage caused by C. difficile TcdB, generally confined to the local intestine, is a severe inflammatory intestinal lesion. We sought to explore whether I-Evs can be applied in this condition as a type of anti-inflammatory immunotherapy. I-Evs contain more TGF- $\beta 1$ than intestinal lysates as determined by western blot, which indicated a likely strong immunosuppressive effect. Next, we established a murine local colon infection model to investigate the treatment effect of I-Evs (Figure 3A). As shown in Figure 3B, the survival rate of mice after C. difficile TcdB injection was only $50 \%$, while I-Evs increased the survival rate of mice up to $80 \%$. The intestinal tissues displayed marked leukocyte 
infiltration and sections of glandular structure damage; consistently, histopathological analysis showed only slight leukocyte infiltration and epithelial cell damage after application of I-Evs (Figure 3C, D). Moreover, intestinal epithelial damage, congestion and mucosal oedema were significantly increased in the $\mathrm{C}$. difficile TcdB mice when compared with the control mice (Figure 3E, F), however, less intestinal damage and limited leukocyte infiltration were observed when mice were treated with I-Evs. These findings implied that I-Evs attenuated pathological changes occurring as a result of C. difficile TcdB-induced inflammation, thereby protecting mice from local colon inflammation.

A

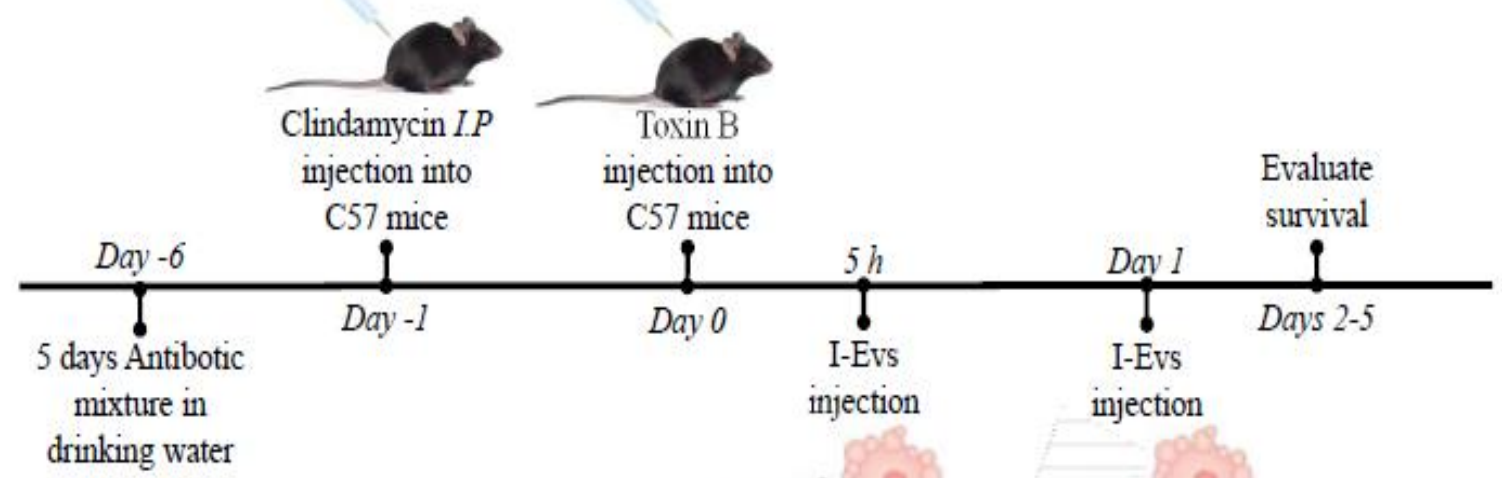

B Survival proportions

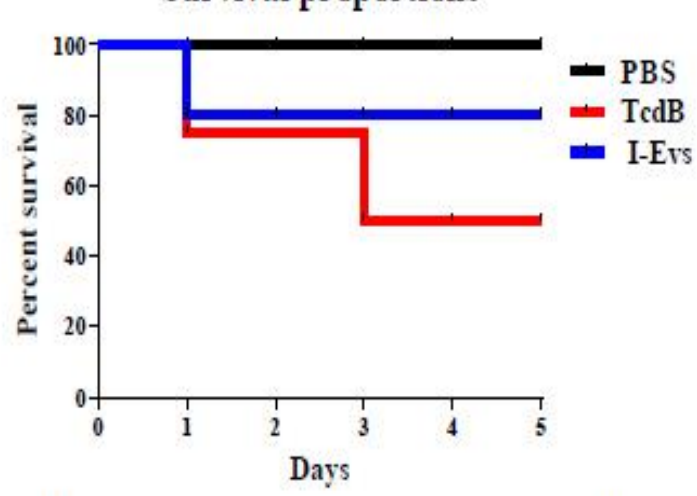

D

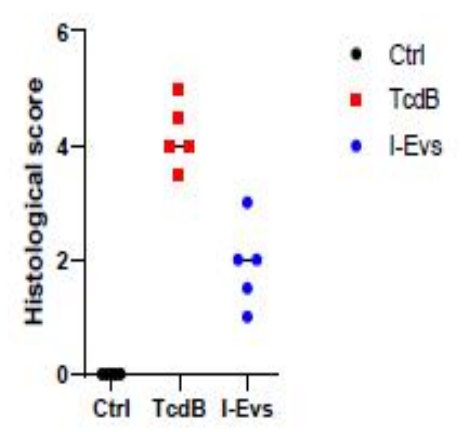

C

Ctrl

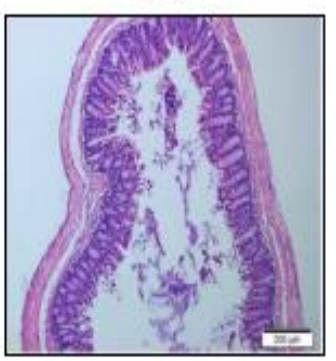

$\operatorname{Tcd} B$

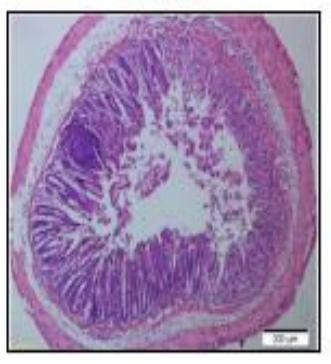

I-Evs

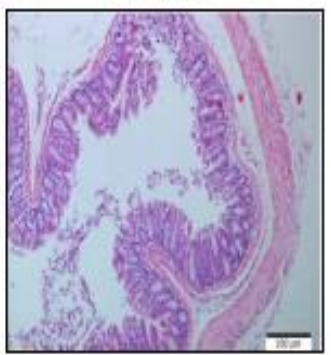

E

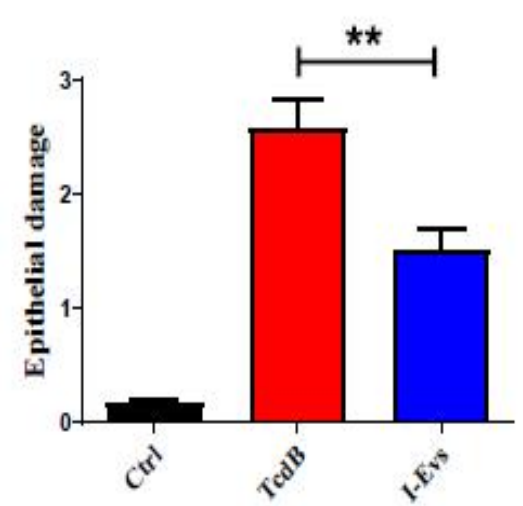

$\mathbf{F}$

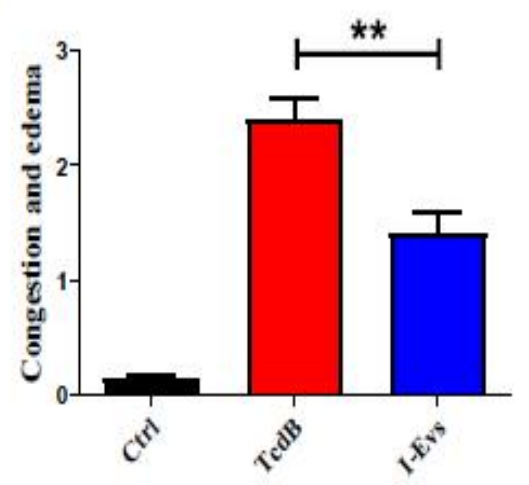

Figure 3: I-Evs alleviate murine C. difficile TcdB-induced local colon inflammation. (A) C57BL/6J mice received antibiotics for 5 days through their drinking water. The antibiotic mixture consisted of gentamicin $(0.035 \mathrm{mg} / \mathrm{mL})$, kanamycin $(0.4 \mathrm{mg} / \mathrm{mL})$, colistin $(850 \mathrm{U} / \mathrm{mL}), \mathrm{metronidazole}$ $(0.215 \mathrm{mg} / \mathrm{mL})$, and vancomycin $(0.045 \mathrm{mg} / \mathrm{mL})$. The following day, mice were injected with clindamycin $(10 \mathrm{mg} / \mathrm{kg})$, followed by C. difficile TcdB $(0.5 \mu \mathrm{g} / \mathrm{kg})$ via surgical injection. This was noted as day 0. Functional I- Evs (50 $\mu \mathrm{g} / 100 \mu \mathrm{L}$ PBS) were injected after $5 \mathrm{~h}$ and on day 1 . (B) The survival rate of mice. $(C)(D)$ Intestinal tissue was collected and prepared for $H \& E$ staining and histological score. Epithelial damage (E) and congestion $(F)$ were scored as histopathological severity. Images are representative of results from five animals, at the indicated time points after the TcdB challenge. All data were verified by three independent experiments. Values represent the mean $\pm S E M(n=5$ animals) versus control animals (*: $P<0.05$, **: $P<0.01$, ***: $P<0.001$, NS: not significant). 
Induction of regulatory $T$ cells by I-Evs alleviated infection caused by $C$. difficile TcdB through a TGF- $\beta 1$-dependent mechanism

A previous study showed that EpCAM-dependent I-Evs alleviated IBD by inducing regulatory $\mathrm{T}$ cells [22]. I-Evs induced an increase in the proportion of CD4+Foxp3+Tregs in vitro and in vivo (Figure 4A-D); these immunoregulatory cells exhibit immunosuppressive effects in the development of disease. When the activity of TGF- $\beta 1$, a potent immunosuppressive cytokine, was blocked (using the protocol described in the Materials and Methods), I-Evs immediately lost the ability to induce CD4+Foxp3+Tregs in the spleen. Concurrently, I-Evs were not able to increase the survival rate of mice, and the improvement of pathological effects previously seen was also undetectable (Figure 4E, F,4G).
A

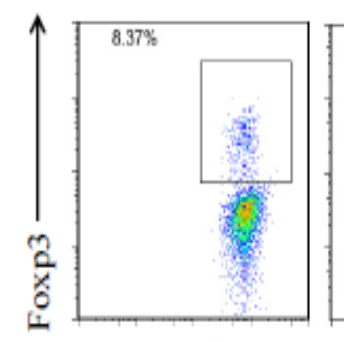

CD4

C

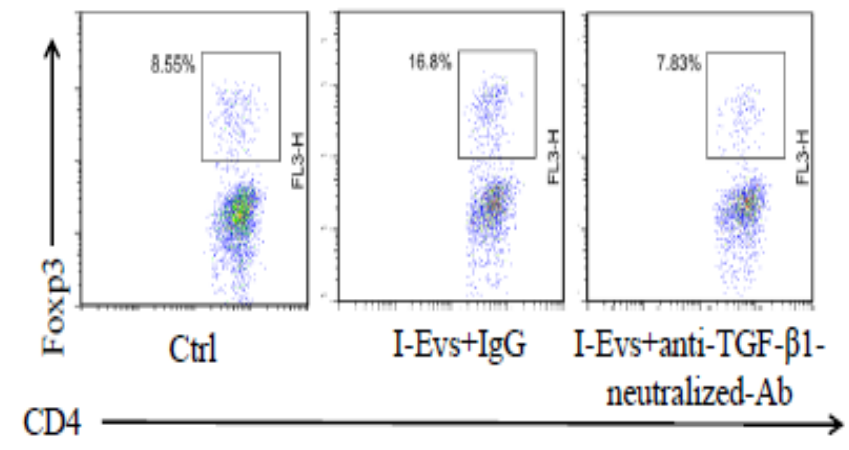

E Survival proportions

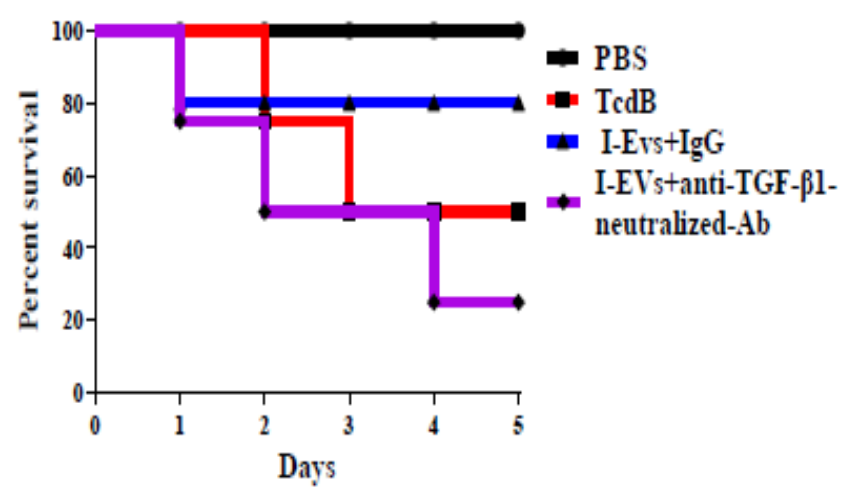

B
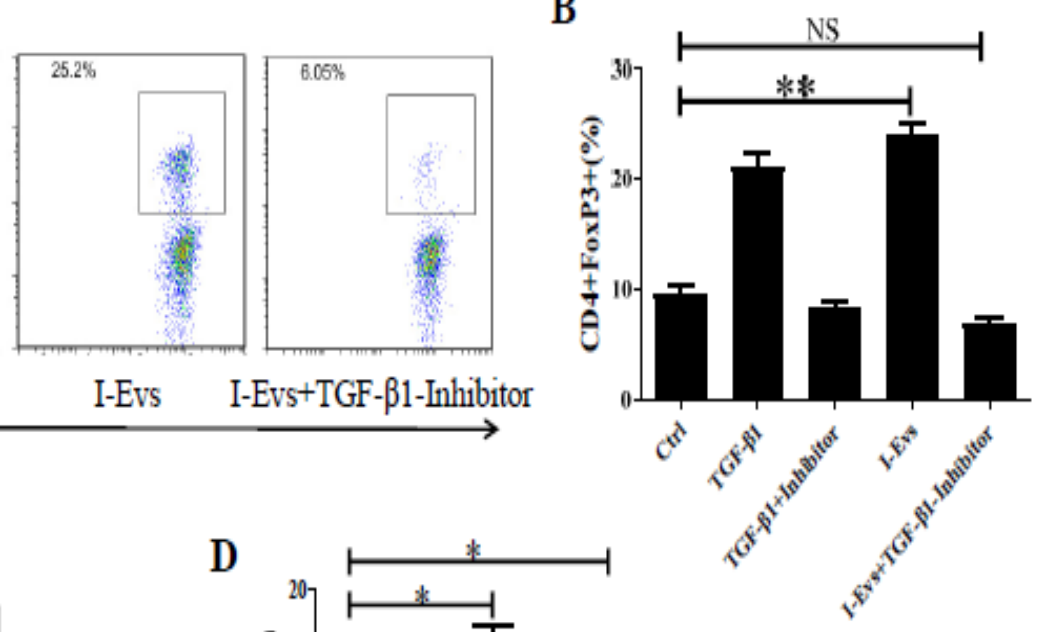

D

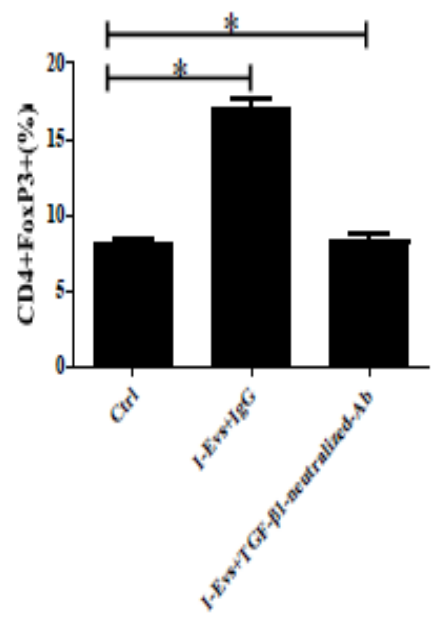


F

Ctrl

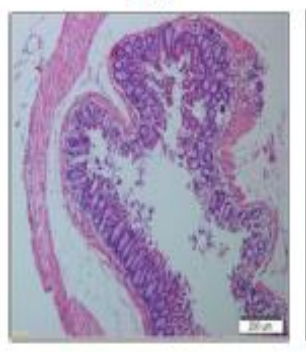

$\operatorname{Tcd} B$

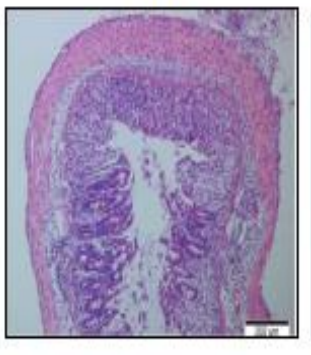

I-Evs+IgG

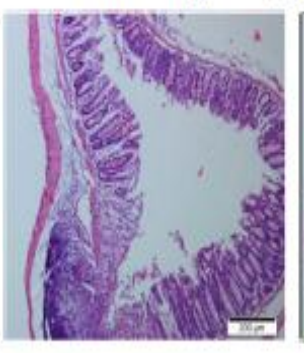

G

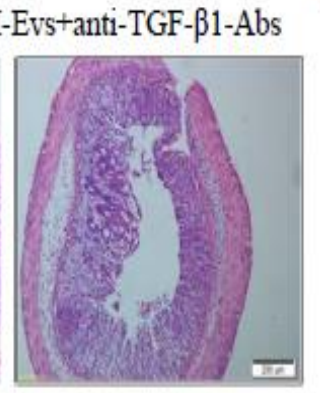

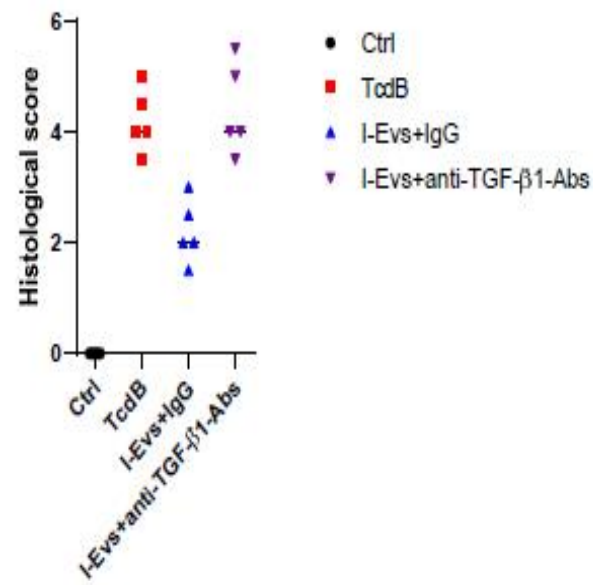

$\mathrm{H}$

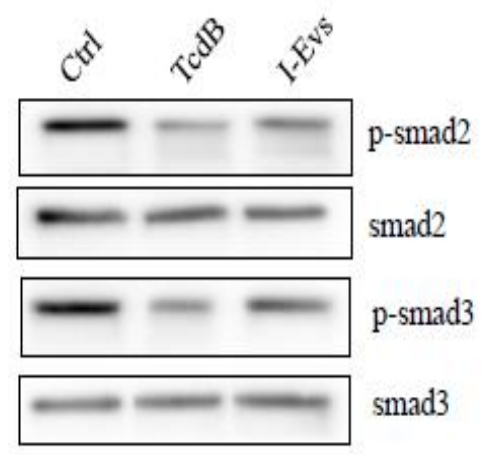

Figure 4: Induction of regulatory T cells by I-Evs alleviated local colon infection caused by $C$. difficile TcdB through a TGF- $\beta 1$-dependent mechanism. (A) A lymphocyte suspension was obtained by grinding and filtering the spleen and lymph nodes of native C57 mice. Naïve CD4 + T cells were magnetically separated with the EasySep Mouse CD4+ T Cell Isolation Kit, and incubated with 200U/mL IL-2 and $\mu$ L anti-CD3/CD28coated beads for $72 \mathrm{~h}(2 \times 105 \mathrm{cell}$ s/well), and separately treated with $3 \mathrm{ng} / \mathrm{mL} T G F-\beta 1,0.6 \mu \mathrm{g} / \mathrm{mL}$ TGF- $\beta 1$ inhibitor, or $50 \mu \mathrm{g} I-E v s$. (B). Statistical analysis of $(A)(n=9)$. (C) To block the TGF- $\beta 1$ signal in vivo, $15 \mu \mathrm{g} / \mathrm{mL}$ anti-TGF- $\beta 1$ - neutralising antibodies were injected into mice and I-Evs were transfused three days later. The percentage of CD4+Foxp + Tregs was analysed by flow cytometry. (D) Statistical analysis of (C) $(n=9)$. (E) The survival rate of mice. $(F)(G)$ The infiltration of neutrophils and destruction of intestinal cells and histological score. $(H)$ Western blot analysis of Smad2/3, and phosphorylated Smad2/3, in MC38 cells stimulated with C. difficile TcdB. All data were verified by three independent experiments. $P$ values were calculated by one-way analysis of variance (ANOVA), versus control animals $(*: P<0.05, * *: P<0.01, * * *: P<0.001$, NS: not significant).

Together, these results suggest that immunosuppressive regulatory T cells induced by I-Evs attenuated $\mathrm{C}$. difficile TcdB- induced local colon inflammation in a mechanism dependent on TGF- $\beta 1$. Smad $2 / 3$ are the main downstream proteins involved in the TGF- $\beta 1$ signalling pathway. The phosphorylation levels of $\mathrm{Smad} 2 / 3$ were decreased after stimulation with C. difficile TcdB, although protein levels of $\mathrm{Smad} 2 / 3$ remained the same; treatment with I-Evs promoted phosphorylation of Smad2/3, and thereby upregulation of TGF- $\boldsymbol{\beta} 1$ (Figure $4 \mathrm{H}$ ). These results suggest that Smad2/3 is inhibited by $\mathrm{C}$. difficile TcdB, leading to the down-regulation of TGF- $\boldsymbol{\beta} 1$ expression. Conversely, I-Evs with high expression of TGF$\boldsymbol{\beta} 1$ activate Smad2/3 and contribute to the upregulation of TGF- $\boldsymbol{\beta} 1$, there by alleviating $\mathrm{C}$. difficile TcdB-induced local colon inflammation in mice.

\section{Discussion}

A common clinical symptom of CDI is local colon infection, which may arise due to intestinal perforation after either infection or intestinal surgery, particularly in high- risk populations, such as patients with IBD; respiratory insufficiency; heart and renal failure; ages over 60 years; and several other underlying diseases. The majority of CDI can be treated with metronidazole and fidaxomicin, in addition to other antibiotics. Surgical removal of necrotic intestinal tissue can reduce mortality rates with severe explosive colitis. Nevertheless, postoperative bleeding, and intestinal stenosis and obstruction, are extremely distressing to the patient. Prevention, management, and non- surgical treatment are the fundamental principles of CDI. However, the most severe toxic colitis cases are unable to benefit from drugs and surgery, and there is an urgent need to establish 
an effective treatment programme based on immunotherapy. Evs participate in a variety of physiological and pathological processes, including neurological disorders [23], osteoarthritis [24], infection [25], and tumours [26]. Evs have been proven to be involved in immune regulation and antigen presentation, and our research group demonstrated that Evs derived from intestinal epithelial cells alleviate IBD in mice by inhibiting dendritic cell activation and inducing Tregs [22]. In this study, I-Evs isolated from the intestine, with mean diameters of $100-200 \mathrm{~nm}$ as detected by electron microscopy scanning and Nanoparticle tracking analysis, expressed the characteristic protein markers of Evs, CD63 and TSG101. Enrichment of the immunosuppressive cytokine, TGF- $\beta$ 1, in IEvs inspired us to hypothesise an immunomodulatory function for I-Evs. A recent study verified that Evs derived from human mesenchymal stem cells can relieve colitis by reducing pro-inflammatory responses and increasing anti-inflammatory responses [27]. As is well-established, colitis caused by $\mathrm{C}$. difficile relies on a series of virulence factors, including toxins, which initially target intestinal epithelial cells and subsequently destroys the intestinal membrane integrity. Hosts exposed to intestinal microorganisms trigger immune inflammatory responses. The dominance of either TcdA or TcdB was still controversial in this research field, despite a multi-laboratory follow-up research study pronouncing that $\mathrm{TcdB}$ acts as a critical toxin in colonic epithelial injury and mortality in vivo, whereas TcdA caused inflammation in mice to a small extent [28]. In the work presented here, TcdB induced increased gene expression of IL-6, TNF- $\boldsymbol{\beta}$, IL-1 $\beta$, and IL-22. I-Evs were able to rescue this phenomenon, and interestingly, TGF- $\beta 1$ and IL- 10 gene actually increased upon co-incubation with I-Evs. Moreover, I-Evs could reverse the decreased expression of TGF- $\beta 1$ stimulated by $\mathrm{C}$. difficile TcdB, as detected by western blot analysis of MC38 and LOVO lysates. We also report for the first time that I-Evs can improve survival of mice with local colon inflammation induced by C. difficile TcdB. The mechanism lies in the induction of CD4+Foxp3+Tregs, which play an important role in maintaining immune tolerance and homeostasis; the decline or dysfunction of Tregs has previously been shown to increase intestinal inflammation in IBD mice[29]29. Similarly, CD4+CD25+ Treg cells transferred into hosts ameliorated colitis symptoms. The I-Evs in this study contained TGF- $\boldsymbol{\beta} 1$; IL-10 is also known to be an important immunosuppressive cytokine, but we could barely detect the presence of IL-10 in our isolated I-Evs. Whether IL-10 still performs an important function is unknown. Furthermore, proinflammatory cytokines were undetectable following stimulation with $\mathrm{C}$. difficile TcdB. Indeed, we improved various experimental schemes to optimise the experimental conditions, unfortunately, the corresponding data were still not available. We speculate that the effect of C. difficile TcdB on cells in vitro was different from that in vivo. As for the animal challenge experiment, in order to induce chronic inflammatory intestinal infection, five antibiotic mixtures were fed to mice, in addition to intraperitoneal injection with clindamycin and local injection with C. difficile Tcd B. I-Evs improved both the survival of mice, and intestinal tissue pathological scores, when transferred into mice.

\section{Conclusions}

We firstly demonstrated that I-Evs can alleviate inflammation induced by $\mathrm{C}$. difficile TcdB in vitro and in vivo, and protect against pathological lesions in the animal intestine. Our data indicated that I-Evs could activate the TGF- $\boldsymbol{\beta} 1$ pathway and the downstream proteins Smad 2/3, to alleviate local colon inflammation induced by $\mathrm{C}$. difficile TcdB, providing a novel endogenous candidate for treatment of $\mathrm{C}$. difficile infections.

\section{Methods}

\section{Toxins, antibodies, and reagents}

C. difficile TcdB was gifted from the Tao Liang research group (West Lake University, Hangzhou, China) [30]. Primary antibodies against
CD63 (ab213090), TGF- $\square 1$ (ab8227), GRP94 (ab238126), TSG101 (ab125011), and $\boldsymbol{\beta}$-Actin (ab8227) were from Abcam (Cambridge, MA, USA). PRMT1 (A33) (\#2449), Smad2 (D43B4), Smad3 (C67H9), phospho-Smad2 (Ser465/Ser467) (E8F3R), and phospho-Smad3 (Ser423/425) were from Cell Signalling Technology (Danvers, MA, USA), and the corresponding secondary antibodies were from BBI (Shanghai, China). Fluorescent- labelled antibodies against CD4 (GK1.5) and Foxp3 (PCH101) were from eBioscienc (San Diego, CA, USA).

\section{Real-time fluorescence quantification PCR}

The classic TRIzol (Gibco, USA) method was used to extract RNA, using a reverse transcription kit (TOYOBO) to acquire cDNA. Real-time, fluorescence quantification PCR (qRT-PCR) was performed in a Step One Plus Real Time PCR System (Roche) to detect gene expression. The mouse-specific primers used are shown in Appendix A.

\section{Mouse and cell lines}

The MC38 cell line was purchased from Wuhan Fine Biotech Co., Ltd. (Wuhan, China). The cells were negative for mycoplasma as detected by fluorescence and culture methods. Human LOVO cells were kindly provided by Jia Jing (Hangzhou Medical College, Hangzhou, China). Male C57BL/6J mice (6-8 weeks old) were purchased from Shanghai Laboratory Animal Co., Ltd. (Shanghai, China). The mice were housed in a specific pathogen-free animal facility, and experimental protocols were approved by the Animal Care and Use Committee of Hangzhou Medical College, all animals were treated according to the guidelines for animal experimentation of Hangzhou Medical College in Hangzhou, China. The animal experiments were also performed in accordance with the ARRIVE (Animal Research: Reporting of In Vivo Experiments) guidelines [31].The mice were sacrificed 5 days after anesthetized with intraperitoneal injection chloral hydrate $(375 \mathrm{mg} / \mathrm{kg}$ of body weight)

\section{Isolation and quantification of I-Evs}

Mouse large intestines were surgically extracted and ground in a sufficient volume of PBS. They were then digested with $1 \mathrm{mg} / \mathrm{mL}$ collagenase type II from Clostridium histolyticum (Gibco) for $2 \mathrm{~h}$ at $37^{\circ} \mathrm{C}$. The resulting suspension of intestinal tissue fragments was centrifuged at $400 \mathrm{~g}$ for 10 $\mathrm{min}$, and the supernatant carefully removed for further centrifugation at $10,000 \mathrm{~g}$ for $30 \mathrm{~min}$, to remove larger vesicles. The resulting supernatant was then filtered by a $0.22-\mu \mathrm{m}$ screening and ultracentrifuged at 100,000 $\mathrm{g}$ for $1 \mathrm{~h}$. Crude pellets of I-Evs were washed in sterile PBS and centrifuged at the same speed for an additional $1 \mathrm{~h}$. The harvested I-Evs were resuspended in PBS. A BCA assay was used to detect the concentration of I-Evs (ThermoFisher, Waltham, MA, USA).

\section{Electron microscopy scanning and Nanoparticle tracking analysis}

Suspensions of I-Evs were loaded onto a coated copper grid, and a drop of $2 \%$ phosphotungstic acid added as a negative staining method. The sample was then allowed to dry at room temperature and transferred to a transmission electron microscope (Hitachi H7650, Hitachi, China) to take pictures and record at a voltage of $80 \mathrm{kV}$. To detect size distribution, IEvs were diluted with PBS, and $0.3 \mathrm{~mL}$ analysed by NanoSight Nano instruments (Malvern, UK).

\section{Western blot and flow cytometry analysis}

For western blot analysis, $40 \mu \mathrm{g}$ I -Evs or protein lysates extracted from intestinal tissues were separated by $12 \%$ sodium dodecyl sulphatepolyacrylamide gel electrophoresis (SDS-PAGE), and transferred to a polyvinylidene fluoride (PVDF) membrane (Millipore, Billerica, MA, USA). Membranes were blocked with $5 \%$ milk in phosphate buffered solution-Tween 20 (PBS-T) and incubated with the corresponding primary antibodies at $4{ }^{\circ} \mathrm{C}$ overnight. The next day, membranes were incubated with an HRP-coupled secondary antibody for $1 \mathrm{~h}$ at room 
temperature and scanned using a Canon 4500 imaging system (Shanghai, China). For cytometry analysis, cells were washed with cold PBS and incubated with a fluorescent antibody for $30 \mathrm{~min}$ at $4{ }^{\circ} \mathrm{C}$ in the dark. Cells were analysed by fluorescence-activated cell sorting (BD,Franklin Lakes, NJ, USA).

\section{CD4+Foxp3+Tregs induction assay}

Murine CD4+ T cells were isolated with the EasySep Mouse CD4+ T Cell Isolation Kit (Stemcell), and labelled with an anti-CD62L antibody for flow cytometry. Magnetic sorting was then performed using the EasySep Mouse Biotin Positive Selection Kit (Stemcell). Cells were then incubated with $1 \mu \mathrm{l}$ anti -CD3/CD28-coated beads and 200U/mL IL-2 for $72 \mathrm{~h}(2 \mathrm{x}$ 105 cells/well), with or without $50 \mu \mathrm{g} / \mathrm{mL}$ I -Evs. To block the TGF- $\beta 1$ signal, $0.6 \mu \mathrm{g} / \mathrm{mL}$ TGF- $\boldsymbol{\beta} 1$ inhibitor was applied to cells (in vitro), or 15 $\mu \mathrm{g} / \mathrm{mL}$ anti-TGF- $\boldsymbol{\beta} 1$-neutralising antibody was injected into mice (in vivo). The percentage of CD4+Foxp3+Tregs was analysed by flow cytometry.

\section{Induction and treatment of murine local colon inflammation induced by $C$. difficile Tcd B}

C57BL/6J male mice were randomised into groups and given antibiotics through their drinking water for 5 days. The antibiotic mixture consisted of gentamicin $(0.035 \mathrm{mg} / \mathrm{mL})$, kanamycin $(0.4 \mathrm{mg} / \mathrm{mL})$, colistin $(850$ $\mathrm{U} / \mathrm{mL})$, metronidazole $(0.215 \mathrm{mg} / \mathrm{mL})$, and vancomycin $(0.045 \mathrm{mg} / \mathrm{mL})$ (Sigma-Aldrich, St. Louis, MO, USA). The following day, mice were injected with clindamycin $(10 \mathrm{mg} / \mathrm{kg})$. After this, purified TcdB was surgical injected into local colon of mice $(0.5 \mu \mathrm{g} / \mathrm{kg})$; this was noted as day 0. Functional I-Evs $(50 \mu \mathrm{g} / 100 \mu \mathrm{L}$ PBS $)$ were intraperitoneal injection after $5 \mathrm{~h}$, and on day 1 . After sacrificing the animals, the intestinal tissue in different groups was collected and prepared for H\&E staining.

\section{Statistical analysis}

Data are presented as the mean \pm SEM. Data were compared using a Student's t- test with GraphPad Prism 8 (San Diego, CA, USA). P $<0.05$ was considered statistically significant.

\section{Abbreviations}

C. difficile: Clostridioides difficile; CDI: Clostridioides difficile infection; FMT: Faecal microbiota transplantation; Evs: Extracellular vesicles

\section{Reference}

1. Lessa FC, Mu Y, Bamberg WM, Beldavs ZG, Dumyati GK, Dunn JR, Farley MM, Holzbauer SM, Meek JI, Phipps EC et al: Burden of Clostridium difficile infection in the United States. The New England journal of medicine 2015, 372(9):825-834.

2. Buonomo EL, Petri WA, Jr.: The microbiota and immune response during Clostridium difficile infection. Anaerobe 2016, 41:79-84.

3. Ng J, Hirota SA, Gross O, Li Y, Ulke-Lemee A, Potentier MS, Schenck LP, Vilaysane A, Seamone ME, Feng $\mathrm{H}$ et al: Clostridium difficile toxin-induced inflammation and intestinal injury are mediated by the inflammasome. Gastroenterology 2010, 139(2):542-552, 552.e541-543.

4. Kociolek LK, Gerding DN: Breakthroughs in the treatment and prevention of Clostridium difficile infection. Nature reviews Gastroenterology \& hepatology 2016, 13(3):150-160.

5. Louie TJ, Miller MA, Mullane KM, Weiss K, Lentnek A, Golan Y, Gorbach S, Sears P, Shue YK: Fidaxomicin versus vancomycin for Clostridium difficile infection. The New England journal of medicine 2011, 364(5):422-431.
6. Yang Z, Schmidt D, Liu W, Li S, Shi L, Sheng J, Chen K, Yu H, Tremblay JM, Chen X et al: A novel multivalent, single-domain antibody targeting TcdA and TcdB prevents fulminant Clostridium difficile infection in mice. The Journal of infectious diseases 2014, 210(6):964-972.

7. Raposo G, Stoorvogel W: Extracellular vesicles: exosomes, microvesicles, and friends. The Journal of cell biology 2013, 200(4):373-383 .

8. Colombo M, Raposo G, Théry C: Biogenesis, secretion, and intercellular interactions of exosomes and other extracellular vesicles. Annual review of cell and developmental biology 2014, 30:255-289.

9. Miyake H, Lee C, Chusilp S, Bhalla M, Li B, Pitino M, Seo S, O'Connor DL, Pierro A: Human breast milk exosomes attenuate intestinal damage. Pediatric surgery international 2020, 36(2):155-163.

10. Yang D, Zhang W, Zhang H, Zhang F, Chen L, Ma L, Larcher LM, Chen S, Liu N, Zhao Q et al: Progress, opportunity, and perspective on exosome isolation- efforts for efficient exosomebased theranostics. Theranostics 2020, 10(8):3684-3707.

11. Babuta M, Furi I, Bala S, Bukong TN, Lowe P, Catalano D, Calenda C, Kodys K, Szabo G: Dysregulated Autophagy and Lysosome Function Are Linked to Exosome Production by Micro-RNA 155 in Alcoholic Liver Disease. Hepatology (Baltimore, Md) 2019, 70(6):2123-2141.

12. Cao SQ, Zheng H, Sun BC, Wang ZL, Liu T, Guo DH, Shen ZY: Long non- coding RNA highly up-regulated in liver cancer promotes exosome secretion. World journal of gastroenterology 2019, 25(35):5283-5299.

13. Wang G, Yuan J, Cai X, Xu Z, Wang J, Ocansey DKW, Yan Y, Qian H, Zhang X, Xu W et al: HucMSC-exosomes carrying miR326 inhibit neddylation to relieve inflammatory bowel disease in mice. Clinical and translational medicine 2020, 10(2):e113.

14. Wu H, Fan H, Shou Z, Xu M, Chen Q, Ai C, Dong Y, Liu Y, Nan $\mathrm{Z}$, Wang $\mathrm{Y}$ et al: Extracellular vesicles containing miR-146a attenuate experimental colitis by targeting TRAF6 and IRAK1. International immunopharmacology 2019, 68:204-212.

15. Liu X, Wang L, Ma C, Wang G, Zhang Y, Sun S: Exosomes derived from platelet-rich plasma present a novel potential in alleviating knee osteoarthritis by promoting proliferation and inhibiting apoptosis of chondrocyte via Wnt/ $\beta$ - catenin signaling pathway. Journal of orthopaedic surgery and research 2019, 14(1):470.

16. Williams AM, Dennahy IS, Bhatti UF, Halaweish I, Xiong Y, Chang P, Nikolian VC, Chtraklin K, Brown J, Zhang Y et al: Mesenchymal Stem Cell- Derived Exosomes Provide Neuroprotection and Improve Long-Term Neurologic Outcomes in a Swine Model of Traumatic Brain Injury and Hemorrhagic Shock. Journal of neurotrauma 2019, 36(1):54-60.

17. Boulestreau J, Maumus M, Rozier P, Jorgensen C, Noël D: Mesenchymal Stem Cell Derived Extracellular Vesicles in Aging. Frontiers in cell and developmental biology 2020, $8: 107$.

18. Harrell CR, Miloradovic D, Sadikot R, Fellabaum C, Markovic BS, Miloradovic D, Acovic A, Djonov V, Arsenijevic N, Volarevic V: Molecular and Cellular Mechanisms Responsible for Beneficial Effects of Mesenchymal Stem Cell-Derived Product "Exo-d-MAPPS" in Attenuation of Chronic Airway Inflammation. Analytical cellular pathology (Amsterdam) 2020, 2020:3153891.

19. Wan S, Wang S, Weng L, Zhang G, Lin Z, Fei X, Zhang F, Yang F, Wang J, Cai Z: CD8 $(+) C D 11 c(+)$ Extracellular Vesicles in the Lungs Control Immune Homeostasis of the Respiratory Tract 
via TGF- $\beta 1$ and IL-10. Journal of immunology (Baltimore, Md : 1950) 2018, 200(5):1651-1660.

20. Allan D, Tieu A, Lalu M, Burger D: Mesenchymal stromal cellderived extracellular vesicles for regenerative therapy and immune modulation: Progress and challenges toward clinical application. Stem cells translational medicine 2020, 9(1):39-46.

21. Mileto SJ, Jardé T, Childress KO, Jensen JL, Rogers AP, Kerr G, Hutton ML, Sheedlo MJ, Bloch SC, Shupe JA et al: Clostridioides difficile infection damages colonic stem cells via TcdB, impairing epithelial repair and recovery from disease. Proceedings of the National Academy of Sciences of the United States of America 2020, 117(14):8064-8073.

22. Jiang L, Shen Y, Guo D, Yang D, Liu J, Fei X, Yang Y, Zhang B, Lin Z, Yang F et al: EpCAM-dependent extracellular vesicles from intestinal epithelial cells maintain intestinal tract immune balance. Nature communications 2016, 7:13045.

23. Chung IM, Rajakumar G, Venkidasamy B, Subramanian U, Thiruvengadam M: Exosomes: Current use and future applications. Clinica chimica acta; international journal of clinical chemistry 2020, 500:226-232.

24. Ni Z, Zhou S, Li S, Kuang L, Chen H, Luo X, Ouyang J, He M, $\mathrm{Du} \mathrm{X}$, Chen L: Exosomes: roles and therapeutic potential in osteoarthritis. Bone research 2020, 8:25.

25. Kumar S, Zhi K, Mukherji A, Gerth K: Repurposing Antiviral Protease Inhibitors Using Extracellular Vesicles for Potential Therapy of COVID-19. Viruses 2020, 12(5).
26. Scavo MP, Depalo N, Tutino V, De Nunzio V, Ingrosso C, Rizzi F, Notarnicola M, Curri ML, Giannelli G: Exosomes for Diagnosis and Therapy in Gastrointestinal Cancers. International journal of molecular sciences 2020, 21(1).

27. Ocansey DKW, Zhang L, Wang Y, Yan Y, Qian H, Zhang X, Xu W, Mao F: Exosome-mediated effects and applications in inflammatory bowel disease. Biological reviews of the Cambridge Philosophical Society 2020, 95(5):1287-1307.

28. Carter GP, Chakravorty A, Pham Nguyen TA, Mileto S, Schreiber F, Li L, Howarth P, Clare S, Cunningham B, Sambol SP et al: Defining the Roles of TcdA and TcdB in Localized Gastrointestinal Disease, Systemic Organ Damage, and the Host Response during Clostridium difficile Infections. mBio 2015, 6(3): 000551.

29. Yamada A, Arakaki R, Saito M, Tsunematsu T, Kudo Y, Ishimaru $\mathrm{N}$ : Role of regulatory $\mathrm{T}$ cell in the pathogenesis of inflammatory bowel disease. World journal of gastroenterology 2016, 22(7):2195-2205.

30. Shen E, Zhu K, Li D, Pan Z, Luo Y, Bian Q, He L, Song X, Zhen Y, Jin D et al: Subtyping analysis reveals new variants and accelerated evolution of Clostridioides difficile toxin B. Communications biology 2020, 3(1):347.

31. Boutron I, Percie du Sert N, Hurst V, Ahluwalia A, Alam S, Avey MT, Baker M, Browne WJ, Clark A, Cuthill IC et al: The ARRIVE guidelines 2.0: Updated
This work is licensed under Creative Commons Attribution 4.0 License
Submit Manuscript
To Submit Your Article Click Here:

DOI: $10.31579 / 2637-8876 / 018$
Ready to submit your research? Choose Auctores and benefit from:

$>$ fast, convenient online submission

$>$ rigorous peer review by experienced research in your field

$>$ rapid publication on acceptance

$>$ authors retain copyrights

$>$ unique DOI for all articles

$>$ immediate, unrestricted online access

At Auctores, research is always in progress.

Learn more https://auctoresonline.org/journals/immunology-and-inflammationdiseases-therapy- 